\section{A Brief Review of Key Models in Cognitive Behaviour Therapy for Psychosis}

Peter Phiri*, Shanaya Rathod, Hannah Carr and David Kingdon

Southern Health NHS Foundation Trust, UK

\section{Abstract}

Cognitive behavioural therapy (CBT) was originally developed as a comprehensive theory for treating depression. It has, however, since been extensively used to treat a wide range of emotional and mental health problems and is now a plausible treatment of choice for schizophrenia and associated psychotic symptoms. This review highlights the different models of CBT used for treating psychosis. These models have varying background, theory and rationale. Overall, literature shows that there are several models and approaches available but an underlying factor shared by all is the centricity of collaborative empiricism and individual formulation of specific presentations, however this varies across models. These models begin to address the impact of culture on treatment of psychosis. That is, they begin to acknowledge that different cultures may have different interpretations of a psychotic presentation such as delusions. It is imperative that models such as those presented here begin to acknowledge cultural influences and take these into account during their formation. This will have an impact on the way psychosis is treated using CBT. By being receptive to this, it could aid the client-therapist relationship and thus the outcomes of treatment.

Keywords: CBT; Psychosis; Cognitive models
*Corresponding author:

Peter Phiri

ఏ peter.phiri@nhs.net peter.phiri@southernhealth.nhs.uk

R\&D Manager, Southern Health NHS Foundation Trust, Tom Rudd Unit, Moorgreen Hospital, Botley Road, West End Southampton, UK.

Tel: 02380475112

Citation: Phiri P, Rathod S, Carr H, Kingdon D (2017) A Brief Review of Key Models in Cognitive Behaviour Therapy for Psychosis. Acta Psychopathol Vol.3 No.S2:84

Received: December 11, 2017; Accepted: December 16, 2017; Published:

December 26, 2017

\section{Introduction}

Schizophrenia has been defined as 'a debilitating illness of the mind, often characterised by symptoms such as hallucinations, delusional beliefs, thought disorder and bizarre behaviours'. Studies of cognitive behavioural therapy (CBT) for psychosis $[1,2]$ demonstrate that if CBT is culturally adapted it would be acceptable to ethnic minority groups [2-4] Models of mental ill health, including cognitive behavioural approaches, have been criticised for being Euro-centric [5] and for their assertion that they are applicable across cultures. Culture significantly impacts on all aspects of psychosis from onset to outcomes.

In the UK, the National Institute for Clinical Excellence (NICE) guidelines recommend CBT as a treatment of choice and put forward that it should be offered to any individual with persistent (positive and negative) symptoms of psychosis and those on remission [6]. Furthermore, the guidelines stipulate that duration of more than 16 planned sessions should be offered, an increase from more than ten sessions previously [7]. Likewise, the American Psychiatric Association [8] practice guidelines recommend this intervention with moderate clinical confidence for schizophrenia.
The current review aims to highlight the models of CBT that are used for treating psychosis. It describes the rationale of these models and how they are used. It aims to also begin to highlight how these models can be adapted and shaped to not only be used on a Euro-centric population but also to diverse cultural populations who require CBT for psychosis.

\section{Theoretical Review}

Theories behind cognitive therapy assume that unhelpful or negative thinking is related to the distress experienced by people; consequently CBT was initially developed as a comprehensive theory of depression [9]. However, it has since been extended to relate to the explanation of a range of other disorders [10] including personality disorders [11] and as an approach to alleviate distress and disturbance associated with psychotic phenomenon [12-15]. Beck described the application of normal cognitive techniques with psychosis to challenge beliefs previously considered nonsusceptible to psychological therapies as early as 1952 [16]. Since then, there was a gradual development in literature of cognitive approaches to psychosis until early 1990s when authors like Chadwick, Birchwood, and Trowler [17] began to investigate 
and publish work on psychosis. The recent advances in cognitive therapy for psychosis emphasis and target emotion [18] worry intervention in psychosis. Notably, the role of attribution biases is implicated in the development and maintenance of psychotic phenomenon in the presence of anomalous experiences.

Cognitive therapy emphasises the role of unhelpful negative ways of thinking in the origin, maintenance and worsening of negative automatic thoughts or thinking errors and rigid depressive schemata. Beck et al. [11] postulate that depressive schemata develop over a period of many years and remain ready to be activated by a combination of stressful circumstances. This early development of negative life experiences causes the formation of beliefs and assumptions/rules about oneself. Consequently cognitive models assert that responses to events are mediated by thoughts and beliefs, that is, personal meaning to them. CBT aims to reduce the symptoms, distress, disturbance and disability associated with a problem (mental, physical or behavioural) and to improve quality of life and wellbeing through cognitive change.

\section{Cognitive Models of Psychosis}

Cognitive models of psychosis suggest that a key factor influencing the psychotic symptoms experienced are the negative interpretations or 'appraisals' of anomalous experiences. Previously, Maher's theory of delusions purports their origin from the application of normal reasoning processes to abnormal experiences [19]. He argues that delusions reflect rational attempts to making sense of anomalous experiences. Simply put, he sees delusions as an explanation. The premise of this model is based on two assumptions. Firstly, on the anomalous experiences which drives a search for meaning, which would be biased by pre-existing beliefs and assumptions about the self, others and the world-view [20]. His second assumption that delusions do not come about through biased reasoning processes has been refuted to date $[21,22]$ and more recently by Coltheart et al., [23] they argue that basic cognitive disturbances are linked to data gathering biases such as a 'jumping to conclusions' (JTC) style of thinking; such patients tend to reach decisions with less evidence resulting in delusional experience. This factor implies bias by deluded individuals in their ability to evaluate their beliefs. This JTC bias and attributional style has continued to be extensively researched. Garety and Freeman [24] have reviewed the literature and continue to support the theory that a JTC bias is associated with the delusions observed within psychosis. These JTC biases also appear to be exuberated during acute states.

Accordingly Coltheart [25] supports the hypothesis that delusional formation involves abnormal reasoning. This model can be sensitive to cultural differences as self-construct can be based on one's culture. Notwithstanding limitations in Maher's second assumption, his theory has impetus in the psychopathology of psychosis.

The evidence to support this hypothesis has expanded beyond that of experimental and self-report data to that of neuroimaging data. In a review by Underwood, Kumari and Peters [26] it has been shown that these biases encompass the amygdala, insula, hippocampus, anterior cingulate cortex, and prefrontal cortex.
Other biases have also been investigated and the recent literature highlighted potentially applicable interpretation and information-processing biases [27]. Though these biases have received some support from the literature this has been limited from experimental studies.

Bentall et al. [28] argue that persecutory beliefs may be the consequence of genuine persecutory experiences, and they note literature that supports particular environmental conditions associated with paranoid thinking, [29-31]. Delusions result from a psychological defence against underlying negative emotion and low self-esteem [32]. An attempt to make sense of anomalous experiences may, according to Bentall, result in 'blaming others' as influenced by the theory of mind deficit. The emphasis on this model is the impact of externalising biases and consideration of sensitive ways to addressing paranoid beliefs.

According to Morrison [14] persecutory delusions are implicated when information is accurately perceived, and then misinterpreted in ways that are culturally unacceptable due to faulty self and social knowledge, influenced by threat beliefs or traumatic experiences rather than faulty perceptions. Therefore their model argues that individuals experience auditory hallucinations following misattribution of intrusive thoughts to an external source in order to reduce the feeling of discomfort resulting from a discrepancy between beliefs and behaviours. Morrison's model draws on cognitive behaviour literature of anxiety disorders. Of particular interest is the emphasis of misinterpretations in the ways that are considered to be culturally unacceptable. This model considers cultural influences, although widely adopted by clinicians its' empirical basis is limited.

\section{Theory of Mind}

Theory of Mind (ToM) refers to an individual's ability to understand that other people have desires and mental states, and explains how individuals comprehend the knowledge and intentions of others.

Frith [33] argued that deficits in ToM could be implicated in the formation of persecutory delusions due to, for example; difficulties arising from monitoring other's thoughts and intentions resulting in paranoid ideation and delusions of reference [34,28].

The model of ToM in relation to psychosis and, in particular, to delusions has been somewhat refuted. In a recent review, Garety and Freeman [24] identified 43 studies of ToM. They argued that the literature shows a clear deficit in ToM in people with Schizophrenia. However, the deficits in ToM were linked more so to negative symptoms as opposed to positive symptoms such as delusions. They thus concluded that although ToM deficits can be identified in patients presenting with delusions they are more strongly associated with the negative symptoms and syndromes.

\section{A multi-factorial model of persecutory delusions}

Freeman \& Garety et al. [35] multifactorial model of the formation and maintenance of persecutory delusions posits that vulnerability to developing psychosis can be explained through bio-psychosocial factors. The model clearly explains the formation and maintenance of psychotic phenomenon with 
onset from significant life events leading to cognitive deficits such as attention, perceptual and jumping to conclusion biases [36]. The authors emphasise the role of emotion in this process in particular threat arousal as a contributory factor to processing of anomalous experiences [35]. Integrating multi-factorial factors in this model makes it ideal for use with diverse cultures as it supports impact of social background in particular traumatic experiences as contributory factors that can exacerbate negative self-schemata and predisposition to psychosis. Furthermore it considers that threat beliefs could result from a number of cognitive biases that the authors implicate in the maintenance cycle such as prejudices that may maintain biased belief systems.

Freeman \& Garety [37] model of persecutory delusions focuses on delusional formation and maintenance. Garety's premise has global applicability as the model posits a cognitive bias that is putatively implicated in the formation of any delusion [38]. The authors posit that any individual with JTC bias from any culture is likely to be influenced by the changing beliefs held by the members of that culture, as reflected by the changes in the content of delusion. Strengths also include normalisation and targets emotion (role of anxiety with psychotic processes is emphasised), through re-evaluation of the threat beliefs and biases in psychological processing explanation [35]. Furthermore, it addresses reasoning and attention biases including safety behaviour and avoidance $[39,40]$. The diagrammatic presentation of this model can be shared with patients to help them make sense of their presenting problems; of particular interest here is the ability to consider possible plausible explanations.

Gumley and Schwannauer [41] cognitive interpersonal approach to recovery identifies how psychotic experiences express developmental adaptation to the critical events and transitions. Attachment theory informs this model through attachment insecurity. That is, factors such as a traumatic birth, being rejected by or loss of a parent in childhood all increase the risk of developing psychosis at a later stage of life. The attachment insecurity resulting from the above factors is associated with the use of avoidant coping strategies. These strategies have been shown to correlate with problematic user engagement in treatment and also low levels of help-seeking $[42,43]$. Although clinically relevant in practice, in that this approach $t$ promotes emotional recovery and reduces risk of relapse, the evidence base for CBT in relapse prevention is limited.

\section{Stress-vulnerability model}

Another widely used theory behind CBT for psychosis is based on a stress-vulnerable model initially developed by Zubin \& Spring [44] and further modified by Nuechterlein et al. [45]; it has been effective in enhancing therapeutic rapport. The model proposes that psychotic symptoms occur as a result of a combination of vulnerabilities in the presence of stressful experiences [46]. Therefore, by learning skills and developing alternative ways of dealing with stress, it is likely that the symptoms will abate. However, this theory continues to be challenged by the proponents of the biological theory who advocate for biological vulnerability as a consequence of genetic predisposition in the development of psychosis. This is despite reports from clinicians, service users and researchers that psychosocial factors do influence the development of psychosis [47].
Kingdon \& Turkingdon [46] emphasise that CBT for psychosis is not identical to standard CBT that is used for axis one problems. What's more, Turkington et al., [48] postulate that cognitive therapy techniques are modified in order to deal with some limitations resulting from schizophrenia, thus list four key therapy stages namely: (i) developing a therapeutic alliance based on the patient's perspective, (ii) developing alternative explanations of schizophrenia symptoms, (iii) reducing the impact of positive and negative symptoms and (iv) offering alternatives to the medical model to address adherence p367.

The fundamental premise in psychological frameworks within cognitive therapy is working collaboratively and the development of shared formulations of individual experiences and an experiential approach to modify beliefs and developing alternative explanations of psychotic phenomenon. This is hoped to result in the patient learning new coping strategies or enhancing helpful strategies and weakening the potency of hallucinations through such strategies as reality testing and behavioural experiments in session and in-between session activities. The fundamental premise of CBT for psychosis is to reduce distress associated with psychotic symptoms [49]. Where a patient is asymptomatic therapy may focus on developing social skills and relapse prevention.

\section{Conclusion}

An evaluation of the literature gives the impression that Cognitive models for Psychosis offer a convincing hypothetical framework which is supported by decades of empirical studies and is becoming more attractive to clinicians. In contrast, models of ToM appear to be less effective in explaining the negative symptoms of psychosis and thus may not be as effective to use as a model in treatment.

The cognitive models, though based upon varying theory and rationale, emphasise the centricity of a collaborative empiricism which varies across cultures in particular when working with collectivist cultures and the paternalistic concepts which differ between western and eastern cultures [50]. This should however not dissuade researchers from meticulously scrutinising all models of CBT for psychosis for cultural sensitivity and addressing needs of diverse cultures. Theorists agree in part, as to which factors are implicated in the formation and maintenance of psychosis.

Effective use of these models in clinical settings requires good therapeutic rapport and engagement. When working with diverse cultures, therapist cognisance of culturally derived behaviours, attitudes, beliefs, values and norms is vital for therapy to be sensitive to the individual's culture whether they have assimilated to the host country or are stuck to the culture of the country of origin [51]. By being sensitive to the culture of the client and thus their interpretation of certain psychotic behaviours it may aid rather than hinder both the client-therapist relationship and thus the outcome of treatments.

Overall, this review has shown that there are a number of models available to use when treating psychosis with CBT. Cognitive models, however, appear to be more suitable, based upon the literature, than models of ToM. It has begun to show that 
these models, though Euro-centric in origin, can be adapted to be applicable to a wider population with various cultural backgrounds. The literature, however, is somewhat limited (e.g. Morrison [14] model of persecutory delusions). Further research

\section{References}

1 Rathod S, Phiri P, Harris S, Underwood C, Thagadur M, et al. (2013) Cognitive behaviour therapy for psychosis can be adapted for minority ethnic groups: a randomised controlled trial. Schizophr Res 143: 319-326.

2 Naeem F, Habib N, Gul M, Khalid M, Saeed S, et al. (2016) Brief cognitive behavioural therapy for psychosis (CBTp) for schizophrenia: literature review and meta-analysis. Int J Cogn Ther 9: 73-86.

3 Rathod S, Kingdon D, Phiri P, Gobbi M (2010) Developing culturally sensitive cognitive behaviour therapy for psychosis for ethnic minority patients by exploration and incorporation of service users and health professionals views and opinions. Behav Cogn Psychother 38: 511-533.

4 Naeem F, Saeed S, Irfan M, Kiran T, Mehmood T, et al. (2015) Brief culturally adapted CBT for psychosis (CaCBTp): a randomized controlled trial from a low income country. Schizophr Res 164: 143148.

5 McCulloch A, Ryrie I, Williamson T, St John T (2005) Has the medical model a future? Ment Health Rev 10: 7-15

6 National Institute for Clinical Excellence (2009) Core interventions in the treatment and management of schizophrenia in primary and secondary care (update) National clinical practice guideline number 82. London, NICE.

7 National Institute for Clinical Excellence (2002) Clinical guidelines 1: Schizophrenia: core interventions in the treatment and management of schizophrenia in primary and secondary care. London, National institute for clinical excellence.

8 APA (2004) Practice guideline for the treatment of patients with schizophrenia (2 ${ }^{\text {nd }}$ Edn). Washington DC, American Psychiatric Association Press.

9 Beck AT, Rush AJ, Shaw BF, Emery G (1979) Cognitive therapy of depression. New York, Guildford.

10 Blackburn IM, Twaddle V (1996) Cognitive therapy in action. London, Souvenir Press.

11 Beck AT, Freeman A, Associates (1990) Cognitive therapy of personality disorders. New York, Guildford Press.

12 Kingdon D, Turkington D (1991) Preliminary report: the use of cognitive behavioural therapy and a normalising rationale in schizophrenia. J Nerv Ment Dis 179: 207-211.

13 Tarrier N, Harwood S, Yussof L (1990) Coping strategy enhancement (CSE): a method of treating residual schizophrenic symptoms. Behav Psychother 18: 643-662.

14 Morrison AP (2001) The interpretation of intrusions in psychosis: an integrative cognitive approach to hallucinations and delusions. Behav Cogn Psychother 29: 257-276.

15 Garety PA, Kuipers E, Fowler D, Freeman D, Bebbington BE (2001) A cognitive model of the positive symptoms of psychosis. Psychol Med 31: 189-195. is imperative in ensuring that the models of $\mathrm{CBT}$ for psychosis are appropriate for use with the diverse population. By grounding these models in empirical research on cultural diversity this could lead to better outcomes of CBT for psychosis.

16 Beck A (1952) Successful outpatient psychotherapy of a chronic schizophrenic with a delusion based on borrowed guilt. Psychiatry 15: 305-312.

17 Chadwick PDJ, Birchwood M, Trowler P (1996) Cognitive therapy for delusions, voices and paranoia. West Sussex, Wiley.

18 Freeman D, Garety PA, Fowler D, Kuipers E, Beddington PE, et al. (2004) Why do people with delusions fail to choose more realistic explanations for their experiences? an empirical investigation. J Consult Clin Psychol 72: 671-680.

19 Maher BA (1992) Models and methods for the study of reasoning in delusions. Revue Europeenne de Psychologie Appliqee 42: 97-102.

20 Maher B (1988) Anomalous experience and delusional thinking: the logic of explanations. In Ottmanns T \& Maher B (Edn) Delusional Beliefs. John Wiley, New York, pp: 15-33.

21 Garety PA, Hemsley DR (1994) Delusions: investigations into the psychology of delusional reasoning. London: Oxford Univ. Press.

22 Garety PA, Freeman D (1999) Cognitive approaches to delusions: a critical review of theories and evidence. Br J Clin Psychol 38: 113-54.

23 Coltheart M, Longdon R, McKay R (2011) Delusional belief. Annu Rev Psychol 62: 271-298.

24 Garety PA, Freeman D (2013) The past and future of delusions research: from the inexplicable to the treatable. Br J Psychiatry 203: 327-333.

25 Coltheart M (2007) Cognitive neuropsychiatry and delusional belief J Exp Psychol 60: 1041-1062.

26 Underwood R, Kumari V, Peters E (2016) Cognitive and neural models of threat appraisal in psychosis: a theoretical integration. Psychiatry Res 239: 131-138.

27 Savulich G, Shergill S, Yiend J (2012) Biased cognition in psychosis. J Exp Psychol 3: 514-536.

28 Bentall RP, Corcoran R, Howard R, Blackwood N, Kinderman P (2001) Persecutory delusions: A review and theoretical integration. Clin Psychol Rev 21: 1143-1192.

29 Mirowsky J, Ross C (1983) Paranoia and the structure of powerlessness. Am Sociol Rev 48: 228-239.

30 Harris T (1987) Recent developments in the study of life events in relation to psychiatric and physical disorders. In Cooper B (Edn) Psychiatric epidemiology: Progress and prospects, London: CroomHelm, pp: 81- 102.

31 Fuchs T (1999) Life events in late paraphrenia and depression. Psychopathology 32: 60.

32 Hassan F (2011) Paranoid delusions: a review of theoretical explanations. ASEAN J Psychiatry 12: 1

33 Frith CD (1992) The cognitive neuropsychology of schizophrenia. Lawrence Eribaum Associates, Hove.

34 Frith C, Corcoran R (1996) Exploring theory of mind in people with schizophrenia. Psychol Med 26: 521-530. 
35 Freeman D, Garety PA, Kuipers E, Fowler D, Beddington P (2002) A cognitive model of persecutory delusions. Br J Clin Psychol 41: 331-347.

36 Garety PA, Hemsley DR, Wessely S (1991) Reasoning in deluded schizophrenic and paranoid patients: biases in performance on a probabilistic inference task. J Nerv Ment Dis 179: 194-258.

37 Freeman D, Garety PA (2004) Paranoia: the psychology of persecutory delusions. Hove: Psychology Press, Hove.

38 Gold J, Gold I (2012) The truman show delusion: psychosis in the global village. Cogn Neuropsychiatry 17: 455-472.

39 Papageorgiou C, Wells A (2003) An empirical test of a clinical metacognitive model of rumination and depression. Cogn Ther Res 27: 261-273.

40 Dugas MJ, Robichaud M (2007) Cognitive behavioural treatment for generalized anxiety disorder: from science to practice. New York: Routledge.

41 Gumley A, Schwannauer M (2006) Staying well after psychosis. Wiley \& Sons.

42 Tait L, Birchwood M, Trower P (2003) Predicting engagement with services for psychosis: insight, symptoms and recovery style. $\mathrm{Br} \mathrm{J}$ Psychiatry 182: 123-128.

43 Tait L, Birchwood M, Trower P (2004) Adapting to the challenge of psychosis: personal resilience and the use of sealing-over (avoidant) coping strategies. Br J Psychiatry 185: 410-415.

44 Zubin J, Spring B (1977) Vulnerability: a new view on schizophrenia. J Abnorm Psychol 86: 103-126.

45 Nuechterlein KH, Dawson ME, Ventura J, Gitlin M, Subotnik KL, et al. (1994) The vulnerability-stress model of schizophrenic relapse: a longitudinal study. Acta Psychiatr Scand 382: 58-64.

46 Kingdon D, Turkington D (2005) Cognitive therapy of schizophrenia. New York: The Guildford Press.

47 Hammersley P, Bullimore P, Fiddler M, Read J (2008) The trauma model of psychosis. In: Stickley T, Basset $T$ (Edn) Learning about mental health practices. Chichester: Wiley, pp: 401-416.

48 Turkington D, Kingdon D, Weiden PJ (2006) Cognitive behaviour therapy for schizophrenia. Am J Schizophr 163: 365-373.

49 Birchwood M, Trower P (2006) The future of cognitive behavioural therapy for psychosis: not a quasi-neuroleptic. Br J Psych 188: 107-108.

50 Laungani P (2004) Asian perspectives in counselling and psychotherapy. Psychology Press.

51 Rathod S, Kingdon D, Pinninti N, Turkington D, Phiri P (2015) Cultural adaptation of CBT for serious mental illness: a guide for training and practice. Wiley-Blackwell, p: 352. 